\title{
A fast and safe technique for sperm preparation in ICSI treatments within a randomized controlled trial $(\mathrm{RCT})$
}

\author{
Domenico Baldini ${ }^{1 *}$ D, Annamaria Baldini ${ }^{1}$, Erica Silvestris ${ }^{2}$, Giovanni Vizziello $^{1}$, Daniele Ferri ${ }^{1}$ and \\ Damiano Vizziello ${ }^{1,3}$
}

\begin{abstract}
Recently a novel method based on horizontal sperm migration in injection dishes has been introduced as an additional tool for preparation of semen sample in assisted reproductive technology (ART) procedures. In the present study, we evaluated both timing and reproductive outcomes in a randomized controlled study including 1034 intra-cytoplasmic sperm injection (ICSI) procedures followed by fresh embryo transfer. Couples enrolled were divided into two sub-groups, namely conventional swim-up method (Group A), and horizontal sperm migration in injection dishes (Group B).

No significant differences were found between groups with respect to fertilization rate, implantation success, clinical pregnancy outcomes and ongoing pregnancies. On the contrary, both cleavage and blastocyst rates were statistically higher in Group B, suggesting superior efficiency and safety of this innovative technique also including time-saving and cheaper costs as compared to the classical swim-up sperm preparation.

Our data support the interpretation of the horizontal sperm migration as a promising procedure for semen preparation in ART cycles.
\end{abstract}

Keywords: Horizontal sperm migration, ICSI, Reproductive outcomes, Sperm preparation

\section{Introduction}

The human ejaculate is a combination of several constituents such as spermatozoa, epithelial and blood cells also including a mixture of immature and necrotic components involved in the synthesis of reactive oxygen species (ROS). The effects of ROS production with respect to DNA stability include both decondensation and fragmentation damage [1] resulting in an impaired fertilization potential of the semen ([2]; Aitken \& Clarckson, 1987a [3, 4]; ). The damaging mechanism of ROS includes the lipid peroxidation of polyunsaturated fatty acids within the sperms' plasmatic membrane with the final result of a reduction of the fast-rectilinear motility and altered

\footnotetext{
* Correspondence: dbaldini@libero.it

${ }^{1}$ Momò Fertilife Clinic, Bisceglie, Italy

Full list of author information is available at the end of the article
}

morphology [5-7] which, however, are not apparently paralleled by potential damages of the sperm DNA [8-12].

Several Authors also reported how elevations of DNA fragmentation in sperm can induce adverse effects on assisted reproduction outcomes including lower fertilization rate and impaired embryo cleavage and implantation (IR) [13-15]. Among conventional techniques for sperm preparation in ART procedures and cycles, the swim-up technique, based on the active migration of spermatozoa from a pre-washed cell pellet within an overlaying medium in relation to the different spermatic gradient density [16], is currently considered a well-established and efficient method [17].

However, although this conventional technique is largely adopted, it also includes several drawbacks related to the increased cell-to-cell contact within the spermatozoa pellet leading to high ROS production [18] 
due to repeated cell centrifugations and long incubation time for samples. Moreover, other disputed aspects of intra-cytoplasmic sperm injection (ICSI) have been related to controversial outcomes $[19,20]$.

Therefore, considering that both timing of each procedure and safety of the biological samples in order to avoid the risk of samples mixture are critical for the in vitro fertilization (IVF) procedure, we performed an easier and faster approach for the semen management which avoids the centrifugation steps and the potential DNA damage induced by the cellular stress, that is based on the horizontal sperm migration directly in the injection dish [21].

Thus, our study is aimed at verifying the efficiency of this novel sperm preparation technique in comparison with the standard swim-up method in ICSI cycles.

\section{Materials and methods}

\section{Determination of the ideal sample size}

In our randomized controlled trial, the sample size was primarily assessed by a mathematical tip (Cochran's sample size formula). Briefly, we first calculated the ideal sample size from the total number of patients undergoing ART procedures in the geographic area of interest (Southern Italy), which include about 3000 cycles per year. Then, by adopting a $95 \%$ confidence interval level and a 3\% margin of error, we obtained 997 cycles as ideal sample, which is slightly lower than the total number of cycles included in this study. Therefore, by considering two distinct Groups A and B of respectively 498 and 536 couples, the allocation report in this study is 1:1.07.

Our study included patients enrolled at our reproductive centre (Momò Fertilife, Bisceglie, Italy), who were recruited from a large geographic area, mainly southern Italy. Since January 2015 to December 2019, we performed 2539 ART cycles including 1650 with fresh embryo transfers (ET), and 880 with frozen ETs. However, considering as inclusion criteria women $<38$ yrs. (without previous ovarian surgery, endometriosis and/or a premature ovarian failure (POF)), and sperm concentration $>1 \times 10^{6}$ million sperm $/ \mathrm{ml}$ for men, the effective enrolled cycles were 1034, whereas 41 couples denied the consent to the study and additional 1464 couples failed to satisfy the inclusion criteria of the study (Fig. 1).

The couples were randomized into two groups according to the sperm preparation for ICSI. Group A $(n=$ 498) included sperm managed by the conventional swim-up method, whereas Group B $(n=536)$ enrolled patients whose sperms were treated by the horizontal swim-up preparation procedure. The women's age ranged between 24 and 38 years with a mean of $34.09 \pm$ 3.08 years, whereas the male age ranged between 24 and 58 years (Table 1). Geographic derivation of patients was prevalently from Apulia region (Table 1). All patients provided their written consent to the study that was approved by the local Ethical Committe, and were anonymized in our database (MedITEX-IVF).

\section{Ovarian stimulation protocol and oocyte collection}

Females from both groups were conventionally treated with the gonadotropin-releasing hormone $(\mathrm{GnRH})$ antagonist (Cetrotide, Merck Serono) and stimulated with recombinant follicle stimulating hormone (FSH) preparation (GONAL-f, Merck Serono). Thus, oocytes were collected between 34 and $37 \mathrm{~h}$ after the human chorionic gonadotropin (hCG) administration and the oocyte retrieval was performed through a vaginal ovarian pick up (OPU) under ultrasound guidance (VOLUSON S8, GE Healthcare).

After $3 \mathrm{~h}$ from the oocyte retrieval, the cumulusoocyte complexes were exposed to $25 \mathrm{IU} / \mathrm{ml}$ hyaluronidase solution (LifeGlobal Group) to remove the corona radiata by repeated pipetting. The retrieved oocytes were then inspected under a stereomicroscope (Nikon SMZ 1500) and only those in metaphase II (MII)-stage, namely mature eggs, were injected after $40 \mathrm{~h}$ from hCG administration.

\section{Semen preparation procedure}

Semen samples from both groups were collected after a sexual abstinence of approximately 3-5 days in accordance with the World of Health Organization (WHO) 2010 guidelines. The samples were maintained at $37^{\circ} \mathrm{C}$ for $20-30 \mathrm{~min}$ in order to complete the liquefaction process and then evaluated under a phase-contrast microscope (Nikon eclipse E 200), while and $10 \mu \mathrm{l}$ of sperm suspension was recovered to assess the sperm count and motility to complete the preparation procedure.

Seminal fluids from Group A patients, were prepared using the conventional swim-up technique by placing samples in a conical tube and supplementation of the same volume of preparing medium (Global, LifeGlobal) followed by centrifuging at $300 \mathrm{~g}$ for $10 \mathrm{~min}$. Supernatants were then discharged and a volume of $0.2-0.5 \mathrm{ml}$ of preparing medium was added to the pellet. The tube was then placed on a stand and tilted at an angle of $45^{\circ}$ and incubated for $30 \mathrm{~min}$. Further, the supernatant was aspirated by a sterile pipette and transferred into a sterile conic tube.

Seminal fluids from Group B patients, were managed by the horizontal swim-up procedure. Briefly, the ICSI plate preparation included 3 additional $50 \mu \mathrm{l}$ drops of Gmops ${ }^{\circ}$ Vitrolife. The drops were linked through a small culture medium using a stripper pipette (Fig. 2a; b). In relation to the concentration and motility of the sperm sample, 1 to $5 \mu \mathrm{l}$ of ejaculate were then injected in the 


\section{FLOW DIAGRAM}

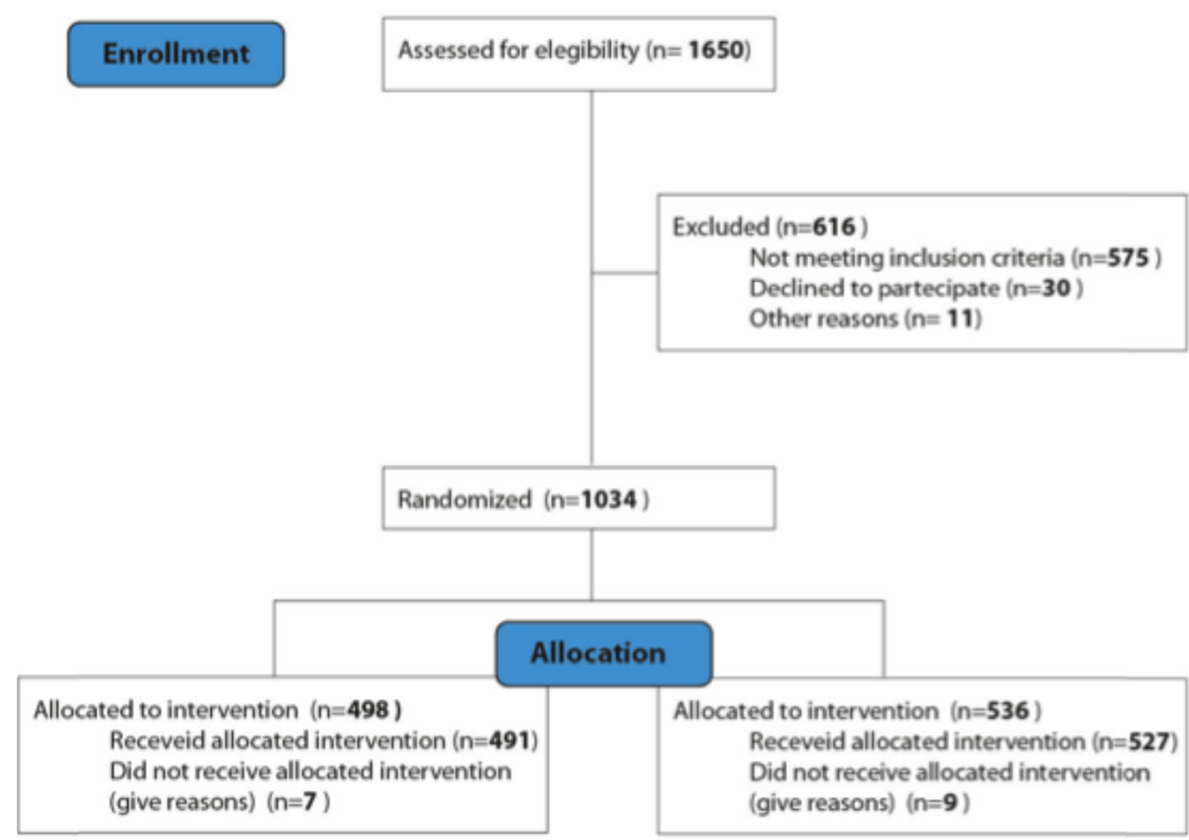

\section{Follow-Up}

\begin{tabular}{|l|l|}
\hline Lost to follow-up (give reasons) $(n=0)$ & Lost to follow-up (give reasons) $(n=0)$ \\
\hline Discontinued intervention (give reasons) $(n=0)$ & Discontinued intervention (give reasons) (n=0) \\
\hline
\end{tabular}

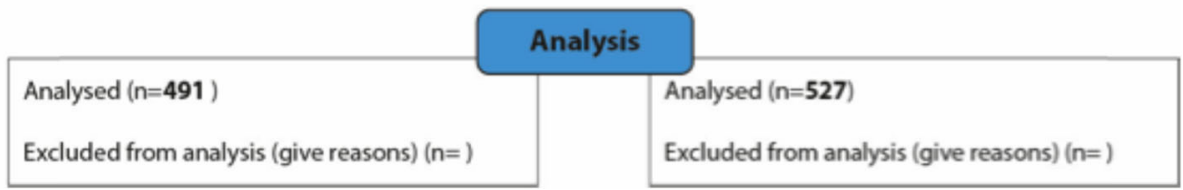

Fig. 1 Flow diagram based on the determination of our sample size used in the study which was split into 2 different groups: A (498) and B (536)

proximal drop $10 \mathrm{~min}$ before presenting oocytes in separated drops (Table 2). During ICSI, adequate amounts of spermatozoa reached the superior edge of the distal drop, and some of them were subsequently recovered by needle and moved in a PVP drop and, subsequently, accurately selected for injection.

\section{Intra-cytoplasmic sperm injection (ICSI) and embryo transfer (ET)}

In both groups, the ICSI procedure was performed at $37^{\circ} \mathrm{C}$ under an inverted microscope (Nikon eclipse TE $200)$ at $400 \mathrm{X}$ magnification. The ICSI was completed by oil-hydraulic assisted microinjection system (Nikon eclipse TE 200). The ET procedure was performed after
3-5 days from OPU by using a catheter (Guardia Access Embryo Transfer Catheter, Cook Medical) with transabdominal ultrasound guidance (General Electric, Logiq V2) for both transfer and implantation of one to two embryos.

\section{Reproductive outcomes}

Fertilization and cleavage rates were evaluated under an inverted microscope (Nikon eclipse TE 200). The presence of 2 pronuclei was assessed 16-18 $\mathrm{h}$ after ICSI and the embryo development was investigated 44-46 h after insemination. The cleavage was evaluated on day 3 . Blood hCG evaluation was performed 12-14 days after ET to assess the pregnancy condition and women with 
Table 1 Demographic and clinical characteristic of the sample

\begin{tabular}{lll}
\hline & A Group $(\boldsymbol{n}=498)$ & B Group $(\boldsymbol{n}=536)$ \\
\hline Female age (years) & $34.07( \pm 3.24)$ & $34.10( \pm 2.93)$ \\
Male age (years) & $36.79( \pm 4.52)$ & $36.9( \pm 3.96)$ \\
Geographical area of origin & & \\
Region & $83 \%$ & $82 \%$ \\
Neighboring regions & $10 \%$ & $11 \%$ \\
Outside the region & $7 \%$ & $7 \%$ \\
Aetiology female infertility & & \\
Tubal & $9,9 \%$ & $10,2 \%$ \\
Reduced ovarian reserve & $25 \%$ & $25,1 \%$ \\
PCOS & $10 \%$ & $9,8 \%$ \\
Various & $10 \%$ & $9,8 \%$ \\
None & $50 \%$ & $50 \%$ \\
Aetiology male infertility & & \\
OA Severe & $22,5 \%$ & $21,4 \%$ \\
OA Moderate & $17,2 \%$ & $17,7 \%$ \\
Various & $11 \%$ & $10 \%$ \\
None & $41,6 \%$ & $43,1 \%$ \\
Years of infertility & & $5,2 \%$ \\
from 1 to 3 & $55,1 \%$ & \\
from 4 to 6 & $28,7 \%$ & \\
from 7 to 9 & $11,7 \%$ & \\
over 9 & $4,5 \%$ & \\
\hline & & \\
& &
\end{tabular}

positive test were monitored 2 weeks later by transvaginal ultrasound examination. Ongoing pregnancies were revealed by the presence of gestational sac and fetal viability at the week 4th after transfer.

\section{Data analysis and statistics}

The randomization frequency was generated by the statistic software STATA 9.0, whereas the allocation sequences were kept hidden to both researchers and the staff in charge of the statistical analysis of samples. A gynaecologist doctor not involved in the treatments, recruited the participants following the mentioned randomization criteria. The study was 'double-blinded' for both couples and researchers. To obtain an accurate statistical assessment of the samples, general characteristics, geographic regions and clinic data such as age, geographic area, ethnicity, reasons and length of infertility, were punctually analysed (Table 1). Mean values of MII oocyte number, oocyte inseminated number and the initial total sperms counts of the two groups were compared (Table 3). Furthermore, data from both groups were evaluated and matched in terms of timing procedures fertilization, cleavage, blastocyst, implantation, clinical pregnancy and ongoing pregnancy rates.
Therefore, data were expressed as mean \pm standard deviation $(\mathrm{M} \pm \mathrm{SD})$ for continuous variables, while percentages were used for categorical variables (Table 3). Mean values were compared by Student's t test. Percentages were compared by Chi-squared test. $P$ value $<0.05$ was considered significant using both statistical analyses. Data analysis was performed by using Statistica version 8.0 (StatSoft Italia Srl, Padova).

\section{Results}

We completed 1034 ICSI cycles with 1898 embryos transferred. ETs were performed in day 3 for both Groups (total number 413) and day 5 (total number 621). In particular, $195 \mathrm{ETs}$ at day 3 and 298 at day 5 were completed in Group A, whereas 218 ETs at day 3 and 323 at day 5, in Group B.

As shown in Table 3, there were no significant differences between the two groups in terms of MII oocyte number, oocyte inseminated number and initially detected sperm concentrations in millions. By contrast, the timing procedures were significantly different between both groups with a relevant time-saving in group B compared to group A (Table 3). Mean values of fertilization, cleavage, blastocyst formation and implantation rates from each group are listed in Table 3 and represented in Fig. 3 (Bar chart 1,2,3). No statistical differences for fertilization rate and implantation rate $(P>0.05)$ were found, whereas both cleavage and blastocyst rates were significantly higher in the group using the novel sperm preparation technique (B). Finally, the clinical pregnancy as well as the ongoing pregnancy rates did not show any statistical differences between the two groups. The results are represented in Table 3 and shown in Fig. 4.

\section{Discussion}

Immediately after ejaculation, sperm is unable to complete fertilization due to the occurrence of immature and necrotic sperm cells as well as blood cells and seminal fluid in the sample, which produces bioactive or toxic endogenous substances. In addition, the collected sperms are exposed to exogenous factors acting as potential sources of ROS, including the effects of visible light, $\mathrm{pH}$ and temperature, as well as centrifugation during the spermatozoa preparation [22].

Therefore, an ideal sperm preparation technique aims to select highly motile, morphologically normal spermatozoa with a minimal DNA damage in a fast, safe and cost-effective manner. Despite several procedures of semen preparation for ART have been developed [17], the well-adopted swim-up technique is unable to provide sperms without DNA damages. In fact, a significant decrease in percentage of normally chromatin-condensed spermatozoa has been reported as recurrent following this procedure [23]. 


\section{a}

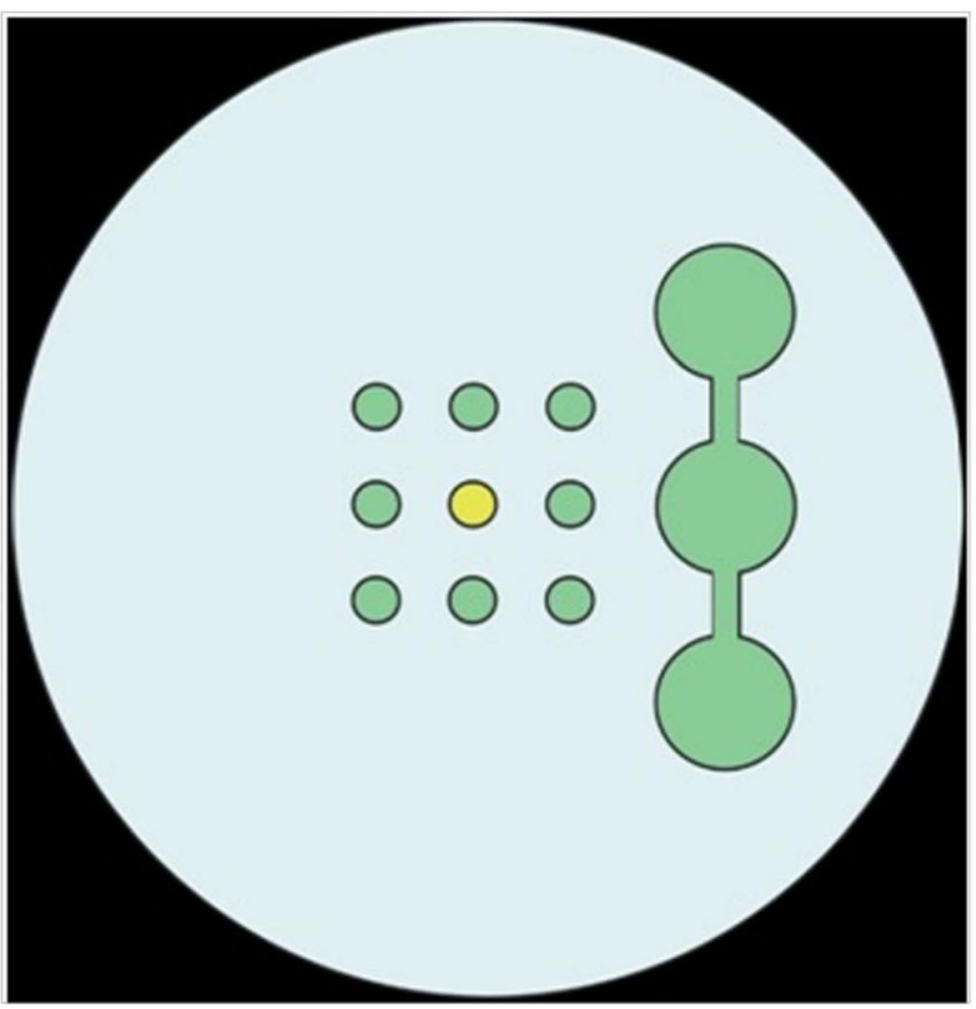

b

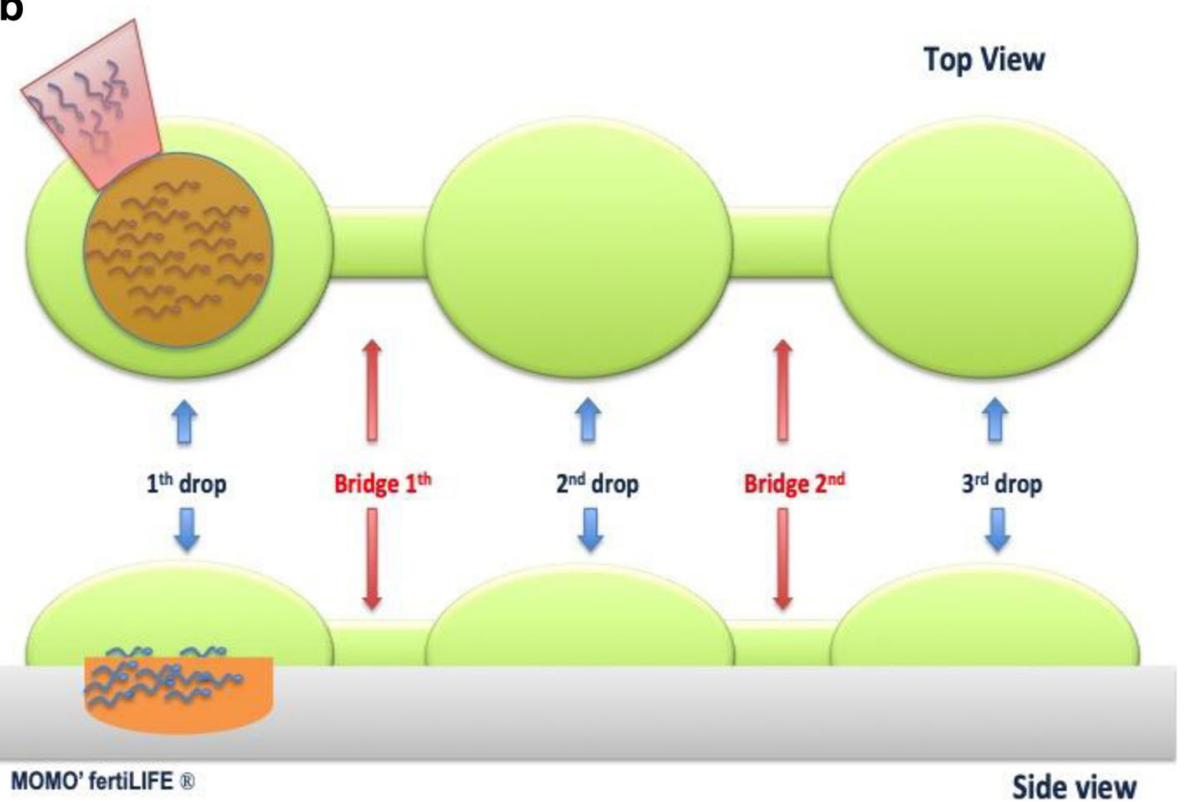

Fig. 2 a ICSI plate scheme. At the centre are schematized culture medium containing oocytes drops (green colour) and PVP drop (yellow colour). On the right are depicted three additional drops linked by small culture medium in which the horizontal swim up is carried out. $\mathbf{b}$ Top and Side viewing of the sperm horizontal migration from the first drop (where the sperms are added) to the third drop (where the sperms are aspirated) trough 2 bridges that link them

Here, we report a simpler and faster procedure for semen preparation based on horizontal sperm migration directly in the injection dish. We compared the ICSI-ET outcomes of this new sperm arrangement with the conventional swim-up technique and found that between the two methods there were no statistical differences in 
Table 2 Quantity of sample's volume in $\mu$ to add in the horizontal migration in relation with concentration $\left(\mathrm{N} \times 10^{6} \mathrm{sptz} / \mathrm{ml}\right)$ and motility $(\% \mathrm{~A}+\mathrm{B})$ of the sperm

\begin{tabular}{llllll}
\hline $\begin{array}{l}\text { Concentration } \\
\text { (N sptz/ml) }\end{array}$ & $>50 \% A+B$ & $50-30 \% A+B$ & $30-20 \% A+B$ & $20-10 \% A+B$ & $10-5 \% A+B$ \\
\hline$\geq 20.000 .000$ & $1 \mu l$ & $1 \mu l$ & $1 \mu l$ & $2 \mu l$ & $3 \mu l$ \\
$20.000 .000-10.000 .000$ & $1 \mu l$ & $1 \mu l$ & $1 \mu l$ & $2 \mu l$ & $3 \mu l$ \\
$10.000 .000-5.000 .000$ & $2 \mu l$ & $2 \mu l$ & $2 \mu l$ & $3 \mu l$ & $3 \mu l$ \\
$5.000 .000-2.000 .000$ & $2 \mu l$ & $2 \mu l$ & $4 \mu l$ & $5 \mu l$ & $5 \mu l$ \\
$2.000 .000-1.000 .000$ & $3 \mu \mathrm{l}$ & $4 \mu \mathrm{l}$ & & $3 \mu \mathrm{l}$ \\
\hline
\end{tabular}

fertilization and implantation rate. Surprisingly, cleavage and blastocyst rates were significantly higher in the group treated with the new method thus implying lower level of fragmented DNA in sperms in accordance with some studies describing that a low DNA Fragmentation Index (DFI) correlates with an increased cleavage rate (Jiang [7, 24]). However, DFI was not investigated in our study. Moreover, it has been reported that embryos obtained from spermatozoa which are exposed to a minor or minimal centrifugation-stress, could have an improved blastocyst development as effect of the major paternal contribution to the development of a normal embryo genome [25]. In line with this observation, we found that both clinical and ongoing pregnancy rates were numerically higher in couples who underwent our innovative approach (group B) although no significant differences were recorded with respect to the group treated with swim-up method (A).

The time saving of the investigated novel technique which is much faster than the classical sperm preparation procedure, is relevant particularly in avoiding the centrifugation steps using density gradients usually requiring up to $20 \mathrm{~min}$, as well as subsequent centrifugations with the culture media to wash the pellet and remove any gradient compound that means more than $10 \mathrm{~min}$, and finally the swim-up from pellet which takes normally between 40 and 50 min [26, 27]. On the contrary, the novel procedure here reported, requires a total of $10 \mathrm{~min}$ to prepare the sperm, with a time saving of about $50 \mathrm{~min}$ compared with the direct swim-up procedure and further 75-80 min when including the density gradient step in the preparation. On the other hand, this procedure is cheaper for avoiding the cost of media that need to be necessarily used with the classical technique of sperm preparation while it has also been demonstrated in the absence of bacterial contaminations [28, 29].

Furthermore, the lower rate of mismatching error between different semen samples of this innovative procedure, should be considered a remarkable aspect to improve processes related to patient identification. However, although in absence of statistically relevant differences, data from direct horizontal sperm migration in injection dishes in terms of pregnancy rate seem to be equal or even better if compared to the conventional procedure while confirming its effectiveness in terms of ICSI outcomes without reducing the fertilization rate [30, 31].

Finally, we would like to emphasize that the statistical assessment was completed in equally distributed groups while a putative weakness of the study is related to the

Table 3 Patient's characteristics and in reproductive outcomes of the two groups included in this study

\begin{tabular}{llll}
\hline & A Group $(\boldsymbol{n}=498)$ & B Group $(\boldsymbol{n}=536)$ & $\boldsymbol{P}$ value \\
\hline Basal sperm concentration $(\times 10[5] / \mathrm{ml})$ & $34.9( \pm 24.65)$ & $36.01( \pm 25.81)$ & 0.67 \\
Retrieved MII oocytes (number) & $5.44( \pm 2.67)$ & $5.6( \pm 3.29)$ & 0.63 \\
Injected MII oocytes (number) & $5.21( \pm 2.46)$ & $5.45( \pm 3.03)$ & $12( \pm 6)$ \\
Timing procedures (minutes) & $61( \pm 12)$ & $78.87 \%( \pm 18.87)$ & 0.42 \\
Fertilization rate & $80.00 \%( \pm 18.35)$ & $98.22 \%( \pm 8.58)$ & 0.0001 \\
Cleavage rate & $92.10 \%( \pm 13.69)$ & $48.1 \%( \pm 18.79)$ & 0.0003 \\
Blastocyst rate & $41.2 \%( \pm 20.69)$ & $24.57 \%( \pm 32.52)$ & 0.0010 \\
Implantation rate & $20.25 \%( \pm 30.90)$ & $209 / 536(38.9 \%)$ & 0.19 \\
Clinical pregnancy/cycle (\%) & $164 / 498(32.8 \%)$ & $172 / 536(32.1 \%)$ & 0.22 \\
Ongoing pregnancy/cycle (\%) & $131 / 498(26.4 \%)$ & & 0.23 \\
\hline
\end{tabular}

Note: Value are expressed as mean \pm sd or percentage 

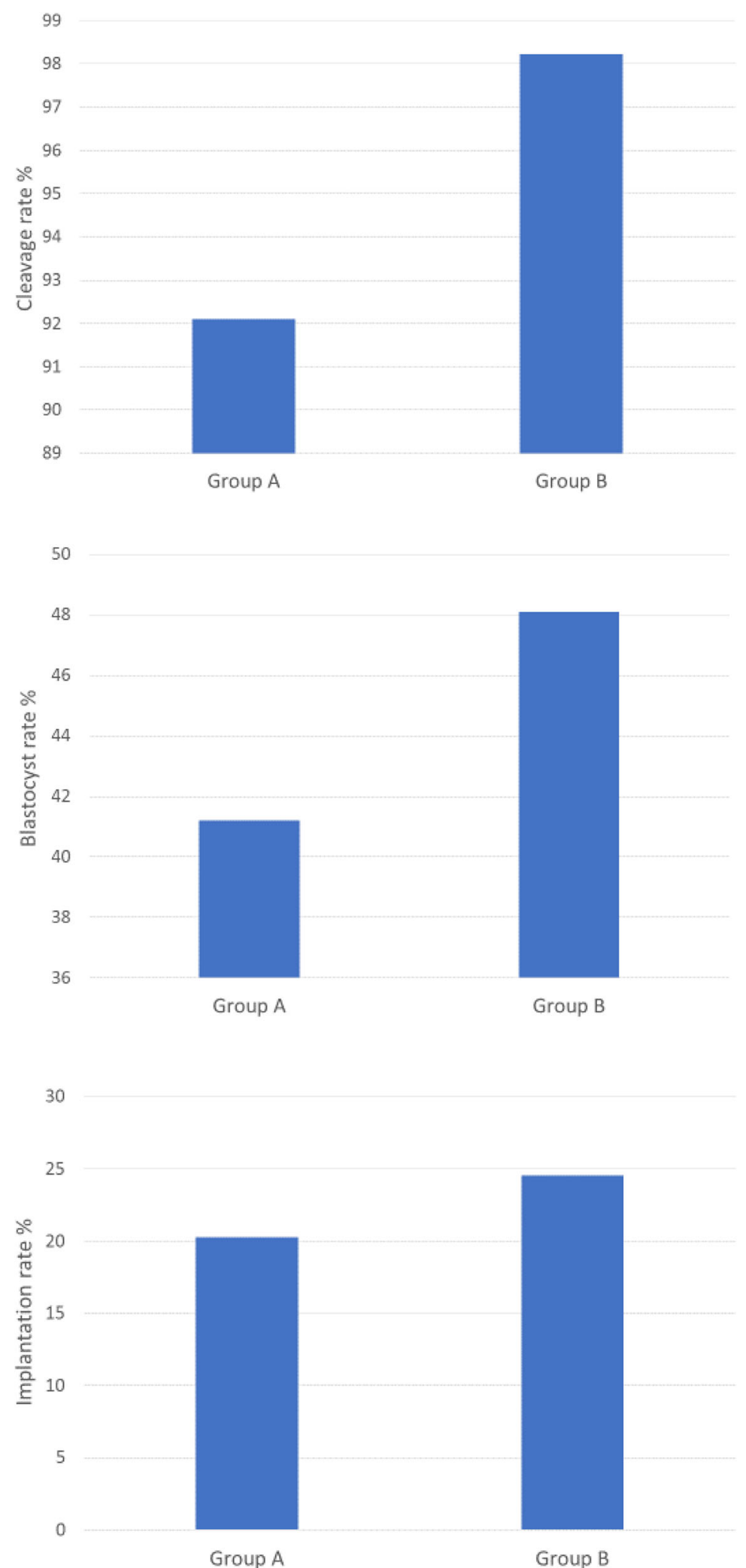

Fig. 3 Bar chart 1 Cleavage rate \% in Group A and Group B; Bar chart 2: Blastocyst rate\% in Group A and group B; Bar chart 3: Implantation rate $\%$ in Group A and Group B 


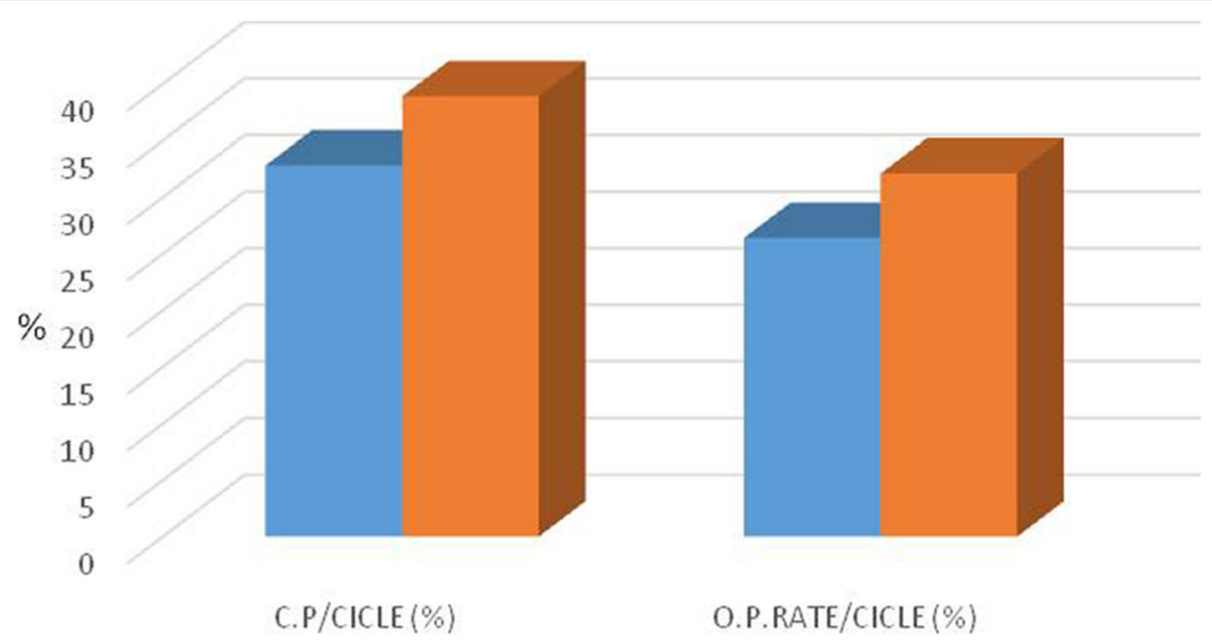

a $\mathrm{A}$ B

Fig. 4 Bar Chart: Clinical Pregnancy Rate and Ongoing Pregnancy Rate in group A and group B

embryo quality, rating as results from data related to the ETs at day 3 and day 5 from both groups. However, data related to both implantation and pregnancy rates of at day 3 and day 5 are not shown since no statistical differences were found.

\section{Conclusion}

In conclusion the horizontal sperm migration directly in the injection dish leads to considerable advantages as it is time-sparing, economical and does not need the involvement of the employed laboratory staff dedicated to the sperm preparation, while allowing to fruitfully work with lower concentration of motile sperms. Based on our favourable data, we plan to apply this method to more patients and to comparatively explore the role of ROS in different techniques of sperm preparations.

\section{Acknowledgements}

Not applicable.

\section{Consent to participate and collection of data}

Data were recorded anonymously by our center database (MedITEX IVF). Informed consent was given to the patients and only those that consented for research purposes were included in this study.

\section{Authors' contributions}

We declare that all the authors have made substantial contributions to all the aspects of the manuscript. We summarized the specific contributions of each author just below: Baldini D.: conception and design of the study; final approval of the version to be submitted Vizziello D.: drafting the article or revising it critically for important intellectual content; Vizziello G: acquisition of data, analysis and interpretation of data Baldini A: acquisition of data, analysis and interpretation of data; Silvestris E: interpretation data, revision of the study and final approval of the version to be submitted; Ferri D: drafting the article and revising it critically for important intellectual content; final approval of the version to be submitted.

\section{Funding}

Funding information is not applicable. No funding was received for the realization of this study.

\section{Availability of data and materials}

The datasets analysed during the current study are available from the corresponding author on reasonable request.

\section{Ethics approval}

The Local Ethics Committee (LEC) of the MOMO' FERTILIFE Center believes that the above research, for the purpose of the study, teaching and training indicated in the project is ethically justified, recommending that the

following conditions to be documented and guaranteed:

- That couples signed the informed consent for the purpose study, research and training;

- That the informed consent has been expressed by an adult, aware and in the absence of any form of material or psychological coercion.

The Opinion in response to the question on the ethical aspects concerning the above research was discussed in the LEC, session of 01/09/2014 and approved unanimously.

Consent for publication

Not applicable.

\section{Competing interests}

All authors declare no competing financial interests or personal relationships potentially responsible to influence the work reported in this paper.

\section{Author details}

${ }^{1}$ Momò Fertilife Clinic, Bisceglie, Italy. ${ }^{2}$ Gynecologic Oncology Unit, IRCCS Istituto Tumori "Giovanni Paolo II", Bari, Italy. ${ }^{3}$ University of Milano, Milan, Italy.

Received: 15 May 2020 Accepted: 6 August 2020

Published online: 19 August 2020

\section{References}

1. Menezo YJ, Silvestris E, Dale B, Elder K. Oxidative stress and alterations in DNA methylation: two sides of the same coin in reproduction. Reprod Biomed Online. 2016;33(6):668-83. 
2. Saleh R, Agarwal A. Oxidative stress and male infertility: from research bench to clinical practice. J Androl. 2002;23:737-52.

3. Aitken RJ, Clarkson JS. Significance of reactive oxygen species and antioxidants in defining the efficacy of sperm preparation technique. $J$ Androl. 1988;9:367-76

4. Aitken RJ, West K, Buckingham D. Leukocytic infiltration into the human ejaculate and its association with semen quality, oxidative stress, and sperm function. J Androl. 1994;15:343-52.

5. Huang C-C, Sc M, David Pei-Cheng Lin PD, Tsao H-M, Sc M, Tzu-Chun Cheng OD, Chung-Hsien Liu MD, Maw-Sheng Lee MD, Ph.D. Sperm DNA fragmentation negatively correlates with velocity and fertilization rates but might not affect pregnancy rates. Fertil Steril. 2005;84:134-5.

6. Saleh RA, Agarwal A, Nada EA, El-Tonsy MH, Sharma RK, Meyer A, Nelson DR, Thomas AJ Jr. Negative effects of increased sperm DNA damage in relation to seminal oxidative stress in men with idiopathic and male factori infertility. Fertil Steril. 2002;79:1602-3.

7. Sun JG, Jurisicova A, Casper RF. Detection of deoxyribonucleic acid fragmentation in human sperm: correlation with fertilization in vitro. Biol Reprod. 1997;56:602-7.

8. Aitken RJ, Smith TB, Lord T, Kuczera L, Koppers AJ, Naumovski N, et al. On methods for the detection of reactive oxygen species generation by human spermatozoa: analysis of the cellular responses to catechol oestrogen, lipid aldehyde, menadione and arachidonic acid. Andrology. 2013;1(2):192-205.

9. Gupta S, Sekhon L, Kim Y, Agarwal A. The role of oxidative stress and antioxidants in assisted reproduction. Current Women's Health Reviews. 2010;6:227-38.

10. Lampiao F. Free radicals generation in an in vitro fertilization setting and how to minimize them. World J Obstet Gynecol. 2012;1:29-34.

11. Moazamian R, Polhemus A, Connaughton H, Fraser B, Whiting S, Gharagozloo P, et al. Oxidative stress and human spermatozoa: diagnostic and functional significance of aldehydes generated as a result of lipid peroxidation. Mol Hum Reprod. 2015;21(6):502-15.

12. Rakhit M, Gokul SR, Agarwal A, du Plessis SS. Antioxidant strategies to overcome OS in IVF-Embryo transfer. In: Studies on Women's Heakth. 2013: 237-62. Humana Press.

13. Benchaib M, Ajina M, Lornage J, Niveleau A, Durand P, Guérin JF. Quantitation by image analysis of global DNA methylation in human spermatozoa and its prognostic value in vitro fertilization: a preliminary study. Fertil Steril. 2003;80:947-53.

14. Lewis SE, Aitken RJ. DNA damage to spermatozoa has impacts on fertilization and pregnancy. Cell Tissue Res. 2005;322:33-41.

15. Lopes S, Sun JG, Jurisicova A, Meriano J, Casper RF. Sperm deoxyribonucleic acid fragmentation is increased in poor-quality semen samples and correlates with failed fertilization in intracytoplasmic sperm injection. Fertil Steril. 1998;69:528-32.

16. Highland HN, Rishika AS, Almira SS, Kanthi PB. Ficoll-400 density gradient method as an effective sperm preparation technique for assisted reproductive techniques. J Human Reprod Sci. 2016;9:194-9.

17. Henkel RR, Schill WB. Sperm preparation for ART. Reprod Biol Endocrinol. 2003;1:108.

18. Takeshima T, Yumura Y, Kuroda S, Kawahara T, Uemura H, Iwasaki A. Effect of density gradient centrifugation on reactive oxygen species in human semen. Syst Biol Reprod Med. 2017;63:192-8. https://doi.org/10.1080/ 19396368.2017.1294214.

19. De Vos A, Van De Velde H, Joris H, Verheyen G, Devroey P, Van Steirteghem A. Influence of individual sperm morphology on fertilization, embryo morphology, and pregnancy outcome of intracytoplasmic sperm injection. Fertil Steril. 2003;79:42-8.

20. Kupker W, al-Hasani S, Schulze W, Kuhnel W, Schill T, Felberbaum R, Diedrich K. Morphology in intracytoplasmic sperm injection: preliminary results. J Assist Reprod Genet. 1995;12:620-6.

21. Vizziello G, Baldini A, Porcelli G, Gliozheni E, Kati K, Baldini D, Vizziello D. A new simple and quick method for sperm preparation and selection prior to ICSI procedure. Curr Trends Clin Embriol. 2015;2:101-2.

22. Agarwal A, Durairajanayagam D, du Plessis SS. Utility of antioxidants during assisted reproductive techniques: an evidence based review. Reprod Biol Endocrinol. 2014;12:112. https://doi.org/10.1186/1477-7827-12-112.

23. Henkel R, Franken DR, Lombard CJ, Schill WB. The selective capacity of glass wool filtration for normal chromatin condensed human spermatozoa: a possible therapeutic modality for male factor cases? J Assist Reprod Genet. 1994;11:395-400
24. Wj J, Jin F, Zhous LM. Influence of the DNA integrity of optimized sperm on the embryionic development and clinical outcomes of in vitro fertilization and embryo transfer. Zhongha Nan Ke Xue (National Journal of Andrology). 2016;22(5):425-31.

25. Simon L, Murphy K, Shamsi MB, Liu L, Emery B, Aston Kl, Hotaling J, Carrell DT. Paternal influence of sperm DNA integrity on early embryonic development. Hum Reprod. 2014;29:2402-12. PMID: 25205757. https://doi. org/10.1093/humrep/deu228.

26. Palini S, Stefani S, Primiterra M, Benedetti S, Barone S, Carli L, Vaccari E, Murat U, Feichtinger W. Comparison of in vitro fertilization outcomes in ICSI cycles after human sperm preparation by density gradient centrifugation and direct micro swim-up without centrifugation. JBRA Assisted Reprod. 2017;21:89-93. https://doi.org/10.5935/1518-0557.20170022.

27. Volpes A, Sammartano F, Rizzari S, Gullo S, Marino A, Allegra A. The pellet swim-p is the best technique for sperm preparation during in vitro fertilization procedures. J Assist Reprod Genet. 2015. https://doi.org/10.1007/ s10815-016-0696-2.

28. Palini S, Primiterra M, De Stefani S, Pedna MF, Sparacino M, Farabegoli P, Benedetti S, Bulletti C, Sambri V. A new micro swim-up procedure for sperm preparation in ICSI treatments: preliminary microbiological testing. JBRA Assisted Reprod. 2016;20:94-8.

29. Henkel R. Sperm preparation: state-of-the-art-physiological aspects and application of advanced sperm preparation methods. Asian J Androl. 2012; 14:260-9. https://doi.org/10.1038/aja.2011.133.

30. Li Z, Zhou Y, Liu R, Lin H, Liu W, Xiao W, et al. Effects of semen processing on the generation of reactive oxygen species and mitochondrial membrane potential of human spermatozoa. Andrologia. 2012;44(3):157-63.

31. Malvezzi H, Sharma R, Agarwal A, Abuzenadah A, Abu-Elmagd M. Sperm quality after density gradient centrifugation with three commercially available media: a controlled trial. Reprod Biol Endocrinol. 2014;12:121. https://doi.org/10.1186/1477-7827-12-121.

\section{Publisher's Note}

Springer Nature remains neutral with regard to jurisdictional claims in published maps and institutional affiliations.

Ready to submit your research? Choose BMC and benefit from:

- fast, convenient online submission

- thorough peer review by experienced researchers in your field

- rapid publication on acceptance

- support for research data, including large and complex data types

- gold Open Access which fosters wider collaboration and increased citations

- maximum visibility for your research: over $100 \mathrm{M}$ website views per year

At BMC, research is always in progress.

Learn more biomedcentral.com/submissions 